\title{
Chronic Recurrent Multifocal Osteomyelitis: A Case Report
}

\author{
Saeed Farzanefar, ${ }^{1}$ Yalda Salehi, ${ }^{1,2,}{ }^{*}$ Mehrshad Abbasi, ${ }^{1}$ and Vahid Ziaee ${ }^{3,4}$ \\ ${ }^{1}$ Department of Nuclear Medicine, Vali-asr Hospital, Tehran University of Medical Sciences, Tehran, IR Iran \\ ${ }^{2}$ Children Medical Center, Pediatric Center of Excellence, Tehran, IR Iran \\ ${ }^{3}$ Rheumatology Research Center, Tehran University of Medical Sciences, Tehran, IR Iran \\ ${ }^{4}$ Department of Pediatrics, Tehran University of Medical Sciences, Tehran, IR Iran \\ "Corresponding author: Yalda Salehi, Department of Nuclear Medicine, Vali-asr Hospital, Tehran University of Medical Sciences, Tehran, IR Iran. Tel: +98-2166575102, Fax: \\ +98-2166581529, E-mail: salehi_y@sina.tums.ac.ir
}

Received 2017 February 23; Revised 2017 July 18; Accepted 2017 August 05.

\begin{abstract}
Introduction: Chronic recurrent multifocal osteomyelitis (CRMO) is a rare migratory skeletal disorder with non-infectious inflammatory etiology which usually causes bone pain in children and adolescents. Intermittent periods of exacerbation and remission are usually noted during the course of the disease. It is a multifocal bone disease usually involving the metaphyses of long bones. The clinical and Paraclinical findings are non-specific, and indeed CRMO is a diagnosis of exclusion based on multiple criteria.

Case Presentation: We present a 6-year-old boy with multiple periods of fever, systemic inflammation and bone pain since he was 2 years old, hospitalized multiple times, received antibiotics and finally diagnosed as a CRMO case.

Conclusions: CRMO should be diagnosed according to a variety of clinical and paraclinical findings. In children and adolescents with multiple bone lesions and lytic lesion, one of the differential diagnoses that should be considered is CRMO.
\end{abstract}

Keywords: Chronic Osteomyelitis, Bone Lesion, FUO, Children

\section{Introduction}

Chronic recurrent multifocal osteomyelitis (CRMO) is an uncommon form of osteitis in children with probably autoimmune origin. It is a rare immigrant inflammatory bone process with intermittent periods of exacerbations and remissions involving multiple sites. Infectious pathogens from affected areas cannot be isolated (1). It was first described by Giedione et al. (2) in 1972 as "an unusual form of multifocal bone lesions with subacute and chronic symmetrical osteomyelitis." Bjorksten and Boquuist(3) understood its relationship with pustulosis and they used the term "chronic recurrent multifocal osteomyelitis."

CRMO is more common in females (4). Lesions typically affect metaphyseal region of long bones but can occur in any bone (such as clavicle) (5-7). Apart from the bone, other organs such as lungs, gastrointestinal tracts and the eyes can also be involved (8). CRMO should be diagnosed with caution. Being aware of the disease helps to preclude the unnecessary use of antibiotic therapy (9). In this report, the case of a 6-year-old with CRMO - which was initially misdiagnosed and treated by multiple courses of different types of antibiotics - is presented.

\section{Case Report}

In 2012, a 2-year-old boy was admitted to the hospital with chief complaint of fever, axial skeleton pain (more in the lumbar region) and difficult walking. Symptoms had been started 2 months prior to the patient's admission. Laboratory investigations at that time showed erythrocyte sedimentation rate (ESR) of 75 and positive C-reactive protein. Conventional X-ray (CXR) did not show any significant finding in favor of infectious process; however, a lytic lesion was noted in the body of L5/S1 in computed tomography (CT) scan. Radiologist suggested the lesion could be due to tumoral involvement or infectious process such as tuberculosis . Other tests including blood culture, PPD, Wright and Coomb's-Wright were all negative. Bone marrow aspiration did not show any specific pathology. Antibiotics therapy (ceftriaxone, clindamycin) was started. According to the radiographic findings, the patient was candidate for open biopsy from lumbar lesion. Moreover, only granulomatous inflammation was reported in pathological evaluation without acid fast bacilli in Ziehl-Nelson staining. Bone culture did not show any organism. Polymerase chain reaction (PCR) analysis was also negative for tuberculosis. In whole body bone scan, multiple foci of increased uptake were noted in the left side of the skull, proximal portion of the left humerus, left femoral neck, distal physis of right femur, L4-L5 vertebrae and posterior arc of the left upper rib. Another bone biopsy from the right femoral lesion was performed and histopathological findings were again in favor of chronic granulomatous inflammation.

In 2014 , he was readmitted to the hospital for more di-

Copyright (c) 2017, Iranian Journal of Pediatrics. This is an open-access article distributed under the terms of the Creative Commons Attribution-NonCommercial 4.0 International License (http://creativecommons.org/licenses/by-nc/4.0/) which permits copy and redistribute the material just in noncommercial usages, provided the original work is properly cited. 
agnostic workup (due to repeated history of bone pain and fever). Bone scintigraphy (Figure 1) revealed new bone lesions in the medial portion of the left clavicle, sacrum, left SI joint, L5 and minimal activity in the left side of the occipital bone. CT scan (Figure 2) showed multiple lytic lesions in the iliac and sacral bones with periosteal reaction. A third bone biopsy from the clavicle lesion was performed and again nonspecific histopathological findings were reported which were compatible with granulomatous inflammation. No organism was obtained from tissue culture, and acid fast staining was negative. The pathologist suggested Eosinophilic granuloma (histiocytosis X) due to histopathological findings. Consequently, the antibiotic therapy was discontinued but due to decreased symptoms the patient refused for more follow up. Two years later on early 2016, the patient was admitted one more time with fever, right arm pain and swelling; besides a necrotic ulcer in the nose was also observed (Figures 3A and 3B). According to nasal ulcer, leprosy was also suspected. Although no sign in favor of skin sensation loss was noted, another biopsy from nasal lesion was performed. Histopathological findings were again in favor of granulomatous inflammation. PCR results and skin smears were negative for leprosy. The patient was referred to our department for bone scan. Bone scintigraphy (Figure 4) revealed increased radiotracer uptake in the right facial bones, right 3rd and 6th ribs, T4, T12, L1, proximal part of left ulna and right iliac bone. ESR was 59 and all other workups including viral marker, flocytometry, immunologic workup, and PPD were unremarkable. The bone scan were compared with previous imaging and considering the immigrant and intermittent nature of the disease CRMO was proposed as the first differential diagnosis and histopathological review of previous biopsies were recommended. Pathological consultation with an expert confirmed the CRMO. Following the diagnosis, prednisolone was started and symptoms subsided. He is now being followed for about 1 year and is still using methotrexate but his symptom is under control.

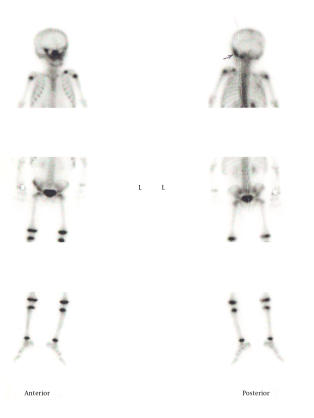

Figure 1. Planar Bone Scan Showing Uptake in the Medial Portion of the Left Clavicle, Sacrum, Left SI Joint, L5 and Minimal Activity in the Left Side of the Occipital Bone

\section{Discussion}

CRMO is a rare skeletal disorder, usually presenting with bone pain and shows intermittent periods of exacerbations and remissions (1). It should be considered as a variant of osteomyelitis (2\% - 5\%) (10) which is thought to have an autoimmune origin. It is more prevalent in females, and usually the mean age of the patients at its onset is about 10 years old $(4,11)$. Patient's symptoms usually continue for several years (median duration is about 6 years). Metaphyseal regions of long bones (distal femur, proximal tibia, distal tibia and distal fibula) are more affected. Other sites of the skeleton which could be involved are clavicles, spine, pelvis and ribs. Mandibular involvement is noted in about $1.5 \%-3 \%$ of the patients with CRMO $(5-7,12-14)$.

Systemic symptoms such as low grade fever and skin lesions can accompany patient's symptoms. Other nonmusculoskeletal disorders related to CRMO include arthritis, palmoplantar pustulosis, Crohn's disease, growth hormone deficiency and psoriasis (15). Elevated ESR is the most remarkable laboratory finding, but anemia and leukocytosis are not detected and also tissue and blood cultures are negative (8).

CRMO may be visible as lytic lesions in the radiography in early phases and later hyperostosis and sclerosis are noted; periosteal reactions can also occur. Also, increased radiopharmaceutical uptake in multiple sites is observed on bone scintigraphy $(1,16)$.

The etiology of CRMO is not well understood. Since the blood and tissue cultures are negative, a noninfectious inflammatory process is the main culprit. Moreover, considering the relationship of CRMO with multiple autoimmune diseases, an autoimmune disorder is also suspected as the cause of the CRMO (17).

In the histopathology of patient with CRMO, the granulomatosis changes may be seen which are noncaseating; Caseous granulomas are mainly seen in TB osteomyelitis. But Assari et al reported a CRMO case that showed caseous granulomas (18).

Some authors have stated that CRMO is the pediatric form of SAPHO syndrome (synovitis, acne, pustulosis, hyperostosis, and osteitis) $(19,20)$.

Different kinds of treatments including non-steroidal anti-inflammatory drugs, corticosteroids, DMARDs, and bisphosphonate can be used (21). Overall, the patients' prognosis is good; however, sometimes the disease persists until adulthood and can cause long term side effects.

CRMO is suspected when repeated episodes of bone pain in different locations occur in a child with some nonspecific findings in plain radiography (which could suggest subacute or chronic osteomyelitis) but without exact histopathological results; also no organism is obtained 

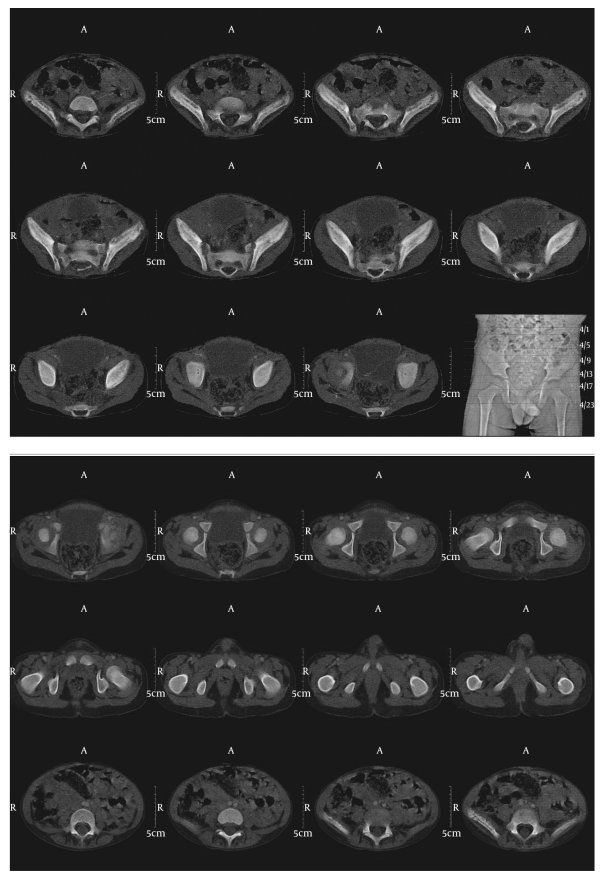

Figure 2. A, Pelvic CT Scan Showing Multiple Lytic Lesions in the Iliac and Sacral Bones with periosteal reaction; B, Pelvic CT Scan Showing Multiple Lytic Lesions in the Iliac and Sacral Bones with Periosteal Reaction
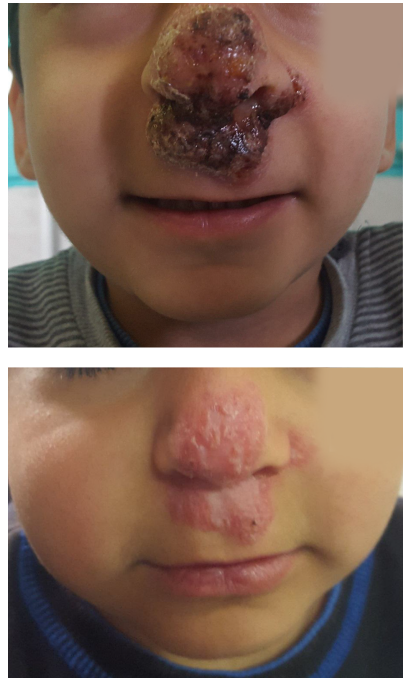

Figure 3. A, Nasal Necrotic Ulcer: Pre-Treatment; B, Nasal Necrotic Ulcer: PostTreatment

from tissue or blood culture.

In this case, the patient had low back pain and fever with lytic lesion in the L5/S1; therefore, tumoral involvement and infectious process such as tuberculosis or brucellosis was considered in differential diagnosis. However,

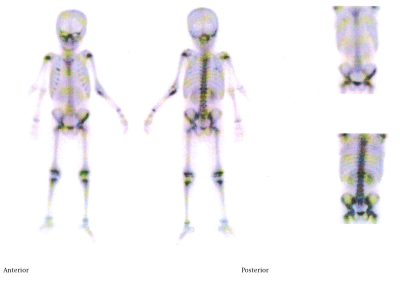

Figure 4. Bone Scan Showing an Increased Radiotracer Uptake in the Right Facial Bones, Right 3rd and 6th Ribs, T4, T12, L1, Proximal Part of Left Ulna and Right Iliac Bone

tissue biopsy did not prove it. According to initial work up, Langerhans cell histocytosis was also suspected, but it was not confirmed on follow up. The interesting point in this case was the patient's nasal ulcer, which - given the fact that he came from an endemic leprosy area - made other causes such as leprosy a plausible scenario. Nasal ulcer biopsy and palatin bone biopsy showed only granulomatous changes. PCR results and skin smears were negative for leprosy. So due to the histopathological findings and patient symptom, leprosy was ruled out. Eventually, based on the patient's history of episodic bone pain involving different sites and bone scan suggestion CRMO was diagnosed and treated accordingly. He is now being followed 
for more than one year and still is using methotrexate, and his symptoms are under control.

\subsection{Conclusion}

CRMO should be diagnosed according to a variety of clinical and para clinical findings. In children and adolescents with multiple bone lesions, one of the differential diagnoses that should be considered is CRMO to prevent multiple bone biopsies and mistreatment.

\section{References}

1. Connolly LP, Drubach LA, Treves ST. In: Nuclear Medicine. Henkin RE, Connolly LP, Drubach LA, Treves T, editors. Pennsylvania: Mosby Elsevier; 2006. pp. 1727-8.Pediatric Skeletal Scintigraphy.

2. Giedion A, Holthusen W, Masel LF, Vischer D. [Subacute and chronic "symmetrical" osteomyelitis]. Ann Radiol (Paris). 1972;15(3):329-42. [PubMed: 4403064].

3. Bjorksten B, Boquist L. Histopathological aspects of chronic recurrent multifocal osteomyelitis. J Bone Joint Surg Br. 1980;62:376-38.

4. Schultz C, Holterhus PM, Seidel A, Jonas S, Barthel M, Kruse K, et al. Chronic recurrent multifocal osteomyelitis in children. Pediatr Infect Dis J. 1999;18(11):1008-13. [PubMed: 10571440].

5. Bjorksten B, Boquist L. Histopathological aspects of chronic recurrent multifocal osteomyelitis. Bone Joint J. 1980;62(3):376-80.

6. Cyrlak D, Pais MJ. Chronic recurrent multifocal osteomyelitis. Skeletal Radiol. 1986;15(1):32-9. [PubMed: 3941921].

7. Jurik AG, Helmig O, Ternowitz T, Moller BN. Chronic recurrent multifocal osteomyelitis: a follow-up study.JPediatr Orthop. 1988;8(1):49-58. [PubMed: 3335622].

8. Alshammari A, Usmani S, Elgazzar AH, Ashkanani RA. Chronic Recurrent Multifocal Osteomyelitis in Children: A Multidisciplinary Approach is needed to establish a Diagnosis. World J Nucl Med. 2013;12(3):120-3. doi: 10.4103/1450-1147.136737. [PubMed: 25165423].

9. Sadeghi E, Kadivar MR, Ghadimi Moghadam AK, Pooladfar GR, Sadeghi N. Chronic recurrent multifocal osteomyelitis: a case report. Iran Red Crescent Med J. 2011;13(1):47-51. [PubMed: 22946018].

10. Girschick HJ, Krauspe R, Tschammler A, Huppertz HI. Chronic recurrent osteomyelitis with clavicular involvement in children: diagnostic value of different imaging techniques and therapy with non-steroidal anti-inflammatory drugs. Eur JPediatr. 1998;157(1):28-33. [PubMed: 9461359].

11. Beretta-Piccoli BC, Sauvain MJ, Gal I, Schibler A, Saurenmann T, Kressebuch H, et al. Synovitis, acne, pustulosis, hyperostosis, osteitis (SAPHO) syndrome in childhood: a report of ten cases and review of the literature. EurJPediatr. 2000;159(8):594-601. [PubMed: 10968238].

12. Probst FP, Bjorksten B, Gustavson KH. Radiological aspect of chronic recurrent multifocal osteomyelitis. Ann Radiol (Paris). 1978;21(2-3):11525. [PubMed: 677736].

13. Jurik AG, Moller BN. Chronic sclerosing osteomyelitis of the clavicle. A manifestation of chronic recurrent multifocal osteomyelitis. Arch Orthop Trauma Surg. 1987;106(3):144-51. [PubMed:3606353].

14. Wipff J, Costantino F, Lemelle I, Pajot C, Duquesne A, Lorrot M, et al. A large national cohort of French patients with chronic recurrent multifocal osteitis. Arthritis Rheumatol. 2015;67(4):1128-37. doi: 10.1002/art.39013. [PubMed: 25545761].

15. Walsh P, Manners PJ, Vercoe J, Burgner D, Murray KJ. Chronic recurrent multifocal osteomyelitis in children: nine years' experience at a statewide tertiary paediatric rheumatology referral centre. Rheumatology (Oxford). 2015;54(9):1688-91. doi: 10.1093/rheumatology/kev013. [PubMed: 25936788].

16. King SM, Laxer RM, Manson D, Gold R. Chronic recurrent multifocal osteomyelitis: a noninfectious inflammatory process. Pediatr Infect Dis J. 1987;6(10):907-11. [PubMed:3696821].

17. Iyer RS, Thapa MM, Chew FS. Chronic recurrent multifocal osteomyelitis: review. AJR Am J Roentgenol. 2011;196(6 Suppl):S87-91. doi 10.2214/AJR.09.7212. [PubMed: 21606238].

18. Assari R, Ziaee V, Ahmadinejad Z, Vasei M, Moradinejad MH. Caseous granuloma: tuberculosis or chronic recurrent multifocal osteomyelitis? Iran J Pediatr. 2014;24(6):770-4. [PubMed: 26019785].

19. Girschick HJ, Raab P, Surbaum S, Trusen A, Kirschner S, Schneider P, et al. Chronic non-bacterial osteomyelitis in children. Ann Rheum Dis. 2005;64(2):279-85. doi: 10.1136/ard.2004.023838. [PubMed: 15647436].

20. Sayili A, Tosun O, Cobanoglu N, Bahceciler Onder N, Baba F, Kavukcu S. Synovitis, Acne, Pustulosis, Hyperostosis, and Osteitis (SAPHO) Syndrome in Childhood; A Rare Clinical Entity. Iran J Pediatr. 2014;24(5):658-9. [PubMed: 25793079].

21. Catalano-Pons C, Comte A, Wipff J, Quartier P, Faye A, Gendrel D, et al. Clinical outcome in children with chronic recurrent multifocal osteomyelitis. Rheumatology (Oxford). 2008;47(9):1397-9. doi 10.1093/rheumatology/ken249. [PubMed: 18621750]. 